\title{
Copy number variation meta-analysis reveals a novel duplication at 9p24 associated with multiple neurodevelopmental disorders
}

Joseph T. Glessner ${ }^{1 \dagger}$, Jin Li ${ }^{1,2+}$, Dai Wang ${ }^{3}$, Michael March', Leandro Lima', Akshatha Desai', Dexter Hadley ${ }^{1}$, Charlly Kao ${ }^{1}$, Raquel E. Gur ${ }^{4}$, Nadine Cohen ${ }^{3}$, Patrick M. A. Sleiman ${ }^{1,5,6}$, Qingqin Li', Hakon Hakonarson ${ }^{1,5,6^{*}}$ and the Janssen-CHOP Neuropsychiatric Genomics Working Group

\begin{abstract}
Background: Neurodevelopmental and neuropsychiatric disorders represent a wide spectrum of heterogeneous yet inter-related disease conditions. The overlapping clinical presentations of these diseases suggest a shared genetic etiology. We aim to identify shared structural variants spanning the spectrum of five neuropsychiatric disorders.

Methods: We investigated copy number variations (CNVs) in five cohorts, including schizophrenia (SCZ), bipolar disease (BD), autism spectrum disorders (ASD), attention deficit hyperactivity disorder (ADHD), and depression, from 7849 cases and 10,799 controls. CNVs were called based on intensity data from genome-wide SNP arrays and CNV frequency was compared between cases and controls in each disease cohort separately. Meta-analysis was performed via a gene-based approach. Quantitative PCR (qPCR) was employed to validate novel significant loci.

Results: In our meta-analysis, two genes containing CNVs with exonic overlap reached genome-wide significance threshold of meta $P$ value $<9.4 \times 10^{-6}$ for deletions and $7.5 \times 10^{-6}$ for duplications. We observed significant overlap between risk CNV loci across cohorts. In addition, we identified novel significant associations of DOCK8/KANK1 duplications (meta $P$ value $=7.5 \times 10^{-7}$ ) across all cohorts, and further validated the CNV region with $\mathrm{qPCR}$.

Conclusions: In the first large scale meta-analysis of CNVs across multiple neurodevelopmental/psychiatric diseases, we uncovered novel significant associations of structural variants in the locus of DOCK8/KANK1 shared by five diseases, suggesting common etiology of these clinically distinct neurodevelopmental conditions.
\end{abstract}

Keywords: Copy number variation, DOCK8, Gene-based analysis, Meta-analysis, Neuropsychiatric disorders, Quantitative PCR

\footnotetext{
* Correspondence: hakonarson@chop.edu

tEqual contributors

${ }^{1}$ The Center for Applied Genomics, Abramson Research Center, Children's

Hospital of Philadelphia, Philadelphia, PA 19104, USA

${ }^{5}$ Department of Pediatrics, Perelman School of Medicine, University of

Pennsylvania, Philadelphia, PA 19104, USA

Full list of author information is available at the end of the article
} 


\section{Background}

Neurodevelopmental and neuropsychiatric disorders represent a wide spectrum of heterogeneous yet interrelated disease conditions with significant overlap in phenotype expression. These diseases in children and young adults pose a major health burden and are growing in prevalence. As the clinical presentations of these diseases are not entirely distinct from each other, the clinical diagnostic boundaries are often hard to define [1].

Genome-wide association studies (GWAS) of single nucleotide polymorphisms (SNPs) in psychiatric diseases have begun to bear intriguing results [1-3]. Copy number variations (CNVs) have more direct gene dosage impacts and have been implicated in psychiatric diseases with larger effect size compared to SNPs [4-7].

Both GWAS and CNV studies have suggested different neuropsychiatric disorders share genetic determinants underlying disease development. It has been estimated that five major psychiatric disorders (schizophrenia (SCZ), bipolar disease (BD), autism spectrum disorders (ASD), attention deficit hyperactivity disorder (ADHD), and depression) share a degree of common genetic etiology. The Psychiatric Genomics Consortium (PGC) study exhibited a certain degree of genetic correlation which is relatively high between SCZ and BD, moderate between $\mathrm{SCZ}$ and depression, moderate between BD and depression, moderate between ADHD and depression, and non-zero between the other pairs of diseases, based on genome-wide SNP genotype data [3]. Common genetic loci have emerged from GWAS focusing on individual neuropsychiatric diseases [2, 8, 9]. Meta-analysis further suggested a shared genetic etiology and the need for molecular diagnostic technology development [1, 10]. Four loci located close to genes ITIH3, AS3MT, $C A C N A 1 C$, and $C A C N B 2$ reached genome-wide significance in meta-analysis of five major psychiatric disorders (SCZ, BD, ASD, ADHD, and depression) in the PGC study with the same direction of effects for these diseases [1]. For three out of the four loci, the five disorders did not show significant difference between each other in a meta-analysis homogeneity test and the model of best fit includes the contribution of all five disorders [1]. An additional seven loci were found approaching genome-wide significance $\left(P\right.$ value $\left.<1 \times 10^{-6}\right)$ and 20 genes in the calcium channel activity pathway were significantly enriched in the dataset of each of the five psychiatric disorders [1]. With the rapid development of high-throughput sequencing technology, a growing cata$\log$ of de novo loss-of-function (LoF) mutations have been identified for each of the neuropsychiatric disorders. Statistical analyses have also revealed significant overlap for LoF mutations between neuropsychiatric disorders [11]. For example, LoF mutations in ten genes are shared between ASD and SCZ (CHD8, ZMYND11, CRYBG3, YTHDC1, HIVEP3, TNRC18, MOV10, ST3GAL6, PHF7, SMARCC2) [11-14]. Compared to the other neuropsychiatric disorders, depression has been more enigmatic. To date, 23 genome-wide significant loci have been identified from five GWAS on major depressive disorder [15-19]. Among these 23 loci, ten (PAX5, RERE, VRK2, MEF2C, L3MBTL2, DCC, SORCS3, NEGR1, VRK2, LIN28B) were shared with other neuropsychiatric disorders (SCZ, BD, ASD, ADHD) reported in the GWAS catalog [20]. Less progress has been made with respect to CNVs. We have observed significant sharing of CNVs across different neurodevelopmental/ psychiatric diseases, impacting genes that belong to the metabotropic glutamate receptor gene networks $[6,7$, 21]; $C A C N A 1 B$ was identified as significant in a schizophrenia case-control CNV study [4], and subsequently a close homolog, CACNA1C, was identified as significant by the PGC GWAS [3]. Deletions upstream of CNTN4 were identified as significant in an autism CNV study [5] as well as significant in an ADHD study [7]. Others have also found common $\mathrm{CNV}$ loci contributing to more than one neuropsychiatric disease. For example, the $\mathrm{CNV}$ at the 16p11.2 locus is associated with both SCZ and BD [22], the duplication at $17 \mathrm{q} 12$ is shared between ASD, intellectual disability, and SCZ [23], and the CNVs at $15 q 13.3,22 q 11.2$, and in the NRXN1 gene are each associated with a wide spectrum of neurodevelopmental disorders [24-26]. However, no study has systematically examined which rare recurrent shared genetic loci bearing CNVs impact the development of multiple neuropsychiatric disorders.

Here, we analyze five major psychiatric disease cohorts, including 7849 cases and 10,799 controls, in a systematic manner to promote comparability of results, and more importantly to understand the degree to which the shared CNV loci may similarly or differently impact the development of neuropsychiatric disorders.

\section{Methods}

\section{Study subjects and genotyping}

Our study is composed of cases of neurodevelopmental and neuropsychiatric diseases and healthy controls from five independent cohorts (Table 1).

\section{Janssen $S C Z$ and $B D$ cohort}

The samples, including 3251 schizophrenia, 377 schizoaffective disorder, and 1344 bipolar cases, were collected from 28 clinical trials conducted by Janssen Research \& Development, LLC, as described previously $[10,27]$. These samples were genotyped on the Illumina $1 \mathrm{MDuoV} 3$ array. They were matched to controls, from the biorepository at the Center for Applied Genomics (CAG) of the Children's Hospital of Philadelphia 
Table 1 The neurodevelopmental and neuropsychiatric disease cohorts analyzed after quality control filtering

\begin{tabular}{llll}
\hline Disease cohort & Cases & Controls & Array \\
\hline Janssen SCZ and BD & 2917 & 1113 & Illumina 1MDv3 \\
CHOP SCZ & 965 & 1467 & Affymetrix 6.0 \\
CHOP ASD & 2079 & 2519 & Illumina 550v3 \\
CHOP ADHD & 1241 & 4110 & Illumina 550v1 \\
Depression & 647 & 1590 & Perlegen 660 k \\
\hline
\end{tabular}

$S C Z$ schizophrenia, $B D$ bipolar disorder, $A S D$ autism spectrum disorders, $A D H D$ attention-deficit hyperactivity disorder

(CHOP), which were also genotyped on the Illumina $1 \mathrm{MDuoV} 3$ arrays to ensure consistency in CNV discovery biases. All controls were recruited at $\mathrm{CHOP}$ and had no diagnosis or family history of psychiatric disease based on their medical record. [10]

\section{CHOP SCZ cohort}

The cases and controls were from CAG at CHOP and the Department of Psychiatry at the University of Pennsylvania, School of Medicine. All cases meet DSM-IV-TR criteria for schizophrenia or schizoaffective disorder [4]. A subject is excluded if he/she is unable to give informed consent to all aspects of the study, or is unable to speak and be interviewed in English, or has severe mental retardation. All samples were genotyped on the Affymetrix 6.0 array at $\mathrm{CHOP}$, as described previously [4].

\section{CHOP ASD cohort}

The autism cohort included 3360 cases from Autism Genetics Resource exchange (AGRE), Autism Genome Project (AGP), and Autism Cases recruited and genotyped at CAG [5]. The control group included children of self-reported Caucasian ancestry, recruited at CHOP. All controls had no history of ASD, or any other central nervous system disorder, chromosomal disorder, syndrome or genetic disorder. All samples were genotyped on the Illumina HumanHap550 chip [5].

\section{CHOP ADHD cohort}

The 1013 ADHD cases of European descent were recruited and genotyped at CHOP; additional cases were from NIMH and The University of Utah [7]. The control group included healthy children aged 6-18 years old, with no serious underlying medical disorder, including but not limited to neurodevelopmental disorders, cancer, chromosomal abnormalities, and known metabolic or genetic disorders [7]. Samples were genotyped on the Illumina HumanHap 550 chip [7].

\section{Depression cohort}

The depression cohort included cases and controls from the Genetic Association Information Network (GAIN) major depressive disorder (MDD)/Netherlands Study of Depression and Anxiety (NESDA) project (phs000020.v2.p1) [28]. The depression cohort cases and controls were genotyped on the Perlegen $660 \mathrm{k}$ array (Perlegen Sciences Mountain View, CA, USA).

\section{Principal component analysis}

PCA was conducted on the SNP genotype using the Eigenstrat [29] package to infer population structure. The first two principal components were plotted to exclude those outliers of non-European ancestry. Only subjects of European ancestry were kept for further association analysis.

\section{CNV detection}

For the Affymetrix 6.0 array, the CEL files were first converted to raw intensity data using our PennCNV [30] Affy workflow (http://penncnv.openbioinformatics.org/en/latest/ user-guide/affy/). Then for all array types, CNVs from all samples were generated using PennCNV [30], a hidden Markov model (HMM)-based algorithm which combines multiple sources of information, including $\log \mathrm{R}$ ratio (LRR), the $\mathrm{B}$ allele frequency (BAF) of each SNP, SNP spacing, and population frequency of $\mathrm{B}$ allele, to generate $\mathrm{CNVs}$. Only $\mathrm{CNVs}$ containing more than three SNPs were generated. As large CNVs tend to be split into small fragments during the CNV calling procedure, adjacent $\mathrm{CNV}$ calls were merged via the clean_cnv.pl program implemented in PennCNV with the default parameters.

\section{Quality control filtering}

Sample quality control (QC) steps were performed to remove the related and/or problematic samples. First, gender discrepancies were examined using both the heterozygosity rate of the X-chromosome SNPs and the call rate of the Y-chromosome SNPs. Samples with discrepant and ambiguous gender information were excluded. Second, the relatedness of the genotyped samples was examined using pairwise Identityby-State analysis via PLINK [31]. Duplicated samples with discrepant phenotype data were excluded from subsequent analyses. For each pair of samples that were duplicates with consistent phenotype data, or samples of related individuals (PI_HAT value exceeding 0.3), the sample with the smaller standard deviation of LRR (LRR SD) was retained.

QC was also conducted on samples based on CNV metrics: genotyping rate, LRR SD indication of intensity noise, |GC base pair wave factor (GCWF)| indication of 
intensity waviness, and CNV count per sample suggestive of DNA quality.

Because of differences between array types, we reviewed data on each array type separately and plotted the distribution of each CNV metric; these metrics typically show a linear phase (the majority of the samples) but can also show an exponential phase (including in samples with outlier values). We excluded subjects with any $\mathrm{CNV}$ metric in the exponential phase. The QC criteria for each cohort based on the distributions of the CNV metrics are shown in (Additional file 1: Table S1).

Next, we performed QC on called CNVs, excluding $\mathrm{CNVs}<20 \mathrm{~kb}$ in length and those encompassing fewer than ten probes.

All QC steps were performed for cases and controls together in each cohort. About $20 \%$ of samples in each cohort were excluded during QC filtering.

\section{Fisher exact test}

Fisher exact test implemented in software ParseCNV [32] was used to conduct the $\mathrm{CNV}$ association analysis on each of the individual cohorts $\mathrm{CHOP} \mathrm{SCZ}, \mathrm{CHOP}$ ASD, CHOP ADHD, and depression. It was also used to compare $\mathrm{CNV}$ frequency between cases and controls, as described in previous publications [5, 32]. Deletions and duplications were analyzed separately.

\section{Linear mixed model association analysis}

The "-includeped" option in ParseCNV [32] was used to generate the ped files for additional CNV analysis using CNV "genotype" status. CNV was converted to "genotype" status in the following way: 11 for $\mathrm{CN}=0,12$ for $\mathrm{CN}=1$, and 22 for others in the deletion ped file; 11 for $\mathrm{CN}=4,12$ for $\mathrm{CN}=3$, and 22 for others in the duplication ped file. Then the CNV ped files were imported into GEMMA version 0.94 [33], which could correct for residual sample structure and population stratification. The LMM association testing was performed on the cohort of Janssen SCZ and BD, the samples of which came from various clinical trials. The relatedness matrix for genotype was calculated using the -gk 1 option. The matrix file was then imported for univariate linear mixed model (LMM) association, and the $-\operatorname{lmm} 4$ option was used. We calculated Wald test, likelihood ratio test, and score test statistics. Then we used InsertPlinkPvalue program from the ParseCNV [32] package to insert the SNP $P$ value generated by GEMMA back into ParseCNV to collapse neighboring SNPs into CNV regions.

\section{CNV annotation}

Because there is no strong consensus in defining a regulatory region and its targeted gene, we focused our analysis on genes with exonic CNVs which are most likely to directly affect the protein product encoded by the genes. The gene(s) that each exonic CNV region resides in was used to annotate each $\mathrm{CNV}$ region. The $\mathrm{CNV}$ association $P$ value from the Fisher exact test or LMM association analysis was assigned to the gene(s). For genes that contain more than one $\mathrm{CNV}$, multiple-testing correction was conducted by taking the lowest $P$ value of the CNVs multiplied by the number of CNVs in this gene.

\section{Meta-analysis}

Fixed effect meta-analysis was then carried out on a gene basis using the software METAL [34]. The logarithm of the odds ratio was taken to ensure consistency with Beta for the direction of association considerations.

\section{Statistical analysis}

We tested a total of 5347 genes harboring exonic CNVs in the meta-analysis of deletions and 6684 genes containing exonic CNVs in the meta-analysis of duplications. Therefore, the multiple-testing adjusted significance threshold is $9.4 \times 10^{-6}$ for deletions and $7.5 \times 10^{-6}$ for duplications. The significance of intersection of risk CNV loci between cohorts was computed using the R package SuperExactTest [35].

\section{CNV quality review}

We conducted manual visual review of the BAF and LRR plots of the significant CNV loci. As we are interested in shared risk genetic loci across different neuropsychiatric diseases, we focused on significant genes harboring case-enriched exonic CNVs in at least two cohorts. BAF and LRR plots of each CNV-containing sample for each significant $\mathrm{CNV}$ region were generated with the visualize_cnv.pl program implemented in the software Penncnv [30].

\section{Quantitative PCR assay}

Quantitative PCR (qPCR) was performed with the Universal Probe Library (UPL). UPL probes (Roche, Indianapolis, IN, USA) and corresponding primers for five assays across the targeted DOCK8/KANK1 region were selected using the ProbeFinder v2.49 software (Roche, Indianapolis, IN, USA). qPCR was conducted on an ABI Prism ${ }^{\text {tw }}$ 7900HT Sequence Detection System (Applied Biosystems, Foster City, CA, USA). For all samples, qPCR reactions were performed in triplicate, each in $10 \mu \mathrm{l}$ of reaction mixture containing $10 \mathrm{ng}$ genomic DNA, $100 \mathrm{nM}$ of the UPL probe, $400 \mathrm{nM}$ of each PCR primer, and 1× TaqMan Gene Expression Master Mix containing UDG and ROX (Life Technologies, Carlsbad, CA, USA), according to the manufacturer's protocol. Male and female genomic DNA (Promega, Madison, WI, USA) were included in the analysis as controls with expected normal copy number. Results were evaluated 
using the Sequence Detection Software v2.4 (Applied Biosystems, Foster City, CA, USA) and further analyzed by the $\Delta \Delta C_{\mathrm{T}}$ method. The GAPDH and SNCA genes were used as internal controls and the geometric mean of their $C_{T}$ values was calculated and used as the reference value for $\Delta \mathrm{C}_{\mathrm{T}}$ calculations. The average of values from Promega male and Promega female genomic DNA (Promega, Madison, WI, USA) was considered the reference $2 \mathrm{~N}$ sample for $\Delta \Delta \mathrm{C}_{\mathrm{T}}$ calculations. Duplications were determined when the relative copy number value for a specific sample normalized to the reference sample was greater than 1.5 .

\section{Results}

To identify shared structural variants underlying the development of neuropsychiatric disorders, we took an unbiased approach based on genome-wide SNP array intensity data from five psychiatric disease cohorts, including SCZ, BD, ASD, ADHD, and depression. After QC filtering based on sample quality and CNV metrics ("Methods"; Additional file 1: Table S1), 7849 cases and 10,799 controls were left for analysis (Table 1). Then we performed case-control association testing on CNVs from each cohort.

Next we performed gene-based meta-analysis. The SNP coverage differs between arrays and one necessary component for $\mathrm{CNV}$ calling, the SNP intensity data, cannot be imputed, so there may not be extensive direct overlap between CNVs across different cohorts. We annotated each $\mathrm{CNV}$ overlapping a gene exon(s) with the gene(s) in which the CNV region resides. Similar to other types of rare variants, CNVs may reside in different regions of a gene, which is important for brain development. Thus, we took a gene-based approach to allow for more dynamic matching between CNVs. The gene-based association testing method has been frequently used in commonvariant and rare-variant analyses [36-42].

In our study, we focused on genes that contain exonic CNVs, which are case-enriched in at least two cohorts. This is because exonic CNVs are most likely to directly affect the protein product encoded by the genes and there is no strong consensus in defining a regulatory region and its targeted gene. Another consideration is the direction of effects that each CNV gives rise to. Though control-enriched CNVs may have potential protective effects, e.g. those of the 22q11.2 CNV region against schizophrenia as reported by Rees et al. [43], this is still actively debated in the CNV study field, and the mechanism by which 22q11.2 CNVs affect the risk of disease development is under investigation. We favor the deleterious model of CNVs, consistent with the field's standard and the focus of our study is risk CNVs shared by neuropsychiatric disorders. Therefore, we kept only significant exonic CNVs that are case-enriched in at least two cohorts. We observed significant overlap of such CNV-containing genes between cohorts (Additional file 2: Figure S1). Two genes (DOCK8 and LOC100131257) were found in all five cohorts $\left(P\right.$ value $\left.=8.29 \times 10^{-6}\right)$.

In the meta-analysis, the ZNF280A and DOCK8 genes reached a genome-wide significance threshold of $9.4 \times$ $10^{-6}$ for deletions and $7.5 \times 10^{-6}$ for duplications (Table 2, Fig. 1) and passed the manual review of their BAF and LRR plots for each $\mathrm{CNV}$ carrier sample (Additional file 2: Figure S2 and S3). KANK1, which is next to $D O C K 8$ and contains exonic duplications, is of suggestive significance $\left(P=3.45 \times 10^{-5}\right)$. The locus of 22q11.22, encompassing the ZNF280A gene, is a known risk CNV locus for SCZ and BD [44]. Deletions overlapping the ZNF280A gene locus were observed in four cohorts. It is significantly case-enriched for the CHOP ADHD and ASD cohorts, in which 22q11.22 deletion was only observed among cases and not among controls, but this locus is control-enriched in the depression and Janssen SCZ and BD cohorts (Table 2, Fig. 1)

We identified a novel CNV locus demonstrating significant association across the neuropsychiatric disorders under study. The DOCK 8 gene at cytoband 9p24.3 showed significant association with neuropsychiatric disorders in meta-analysis. DOCK 8 and the neighboring gene KANK1 exhibited significant or marginally significant case enrichment in all five cohorts (Tables 2 and 3, Fig. 2), especially in the Janssen SCZ and BD cohort as well as the CHOP ASD cohort, in which more than 5 cases but no controls carry CNVs in this region (Table 3). As the samples in the Janssen SCZ and BD cohort were from diverse resources and were not genotyped at $\mathrm{CHOP}$, to ensure $\mathrm{CNV}$ quality at this region, we

Table 2 Significant loci in gene-based meta-analysis of the five neurodevelopmental/neuropsychiatric cohorts that contain caseenriched exonic CNVs in two or more cohorts

\begin{tabular}{llllll}
\hline Marker name & Cytoband & CNV type & Number of cohorts observed & Meta $P$ value & Direction of effect \\
\hline ZNF280A & $22 q 11.22^{*}$ & Del & 4 & $8.63 \mathrm{E}-08$ & $-?++-$ \\
DOCK8 & $9 \mathrm{p} 24.3$ & Dup & 5 & $7.50 \mathrm{E}-07$ & +++++ \\
\hline
\end{tabular}

The Number of cohorts is the number of cohorts in which CNVs were observed overlapping exons of the gene. The Direction of effect is reported in the order of Janssen SCZ and BD, CHOP SCZ, CHOP ASD, CHOP ADHD, and depression cohorts: a plus sign means CNVs are enriched in cases, a dash means CNVs are enriched in controls, a question mark means no CNV is observed overlapping the exons of this gene. Del deletion, Dup duplication.

${ }^{*}$ Known neuropsychiatric-disorder-associated regions 

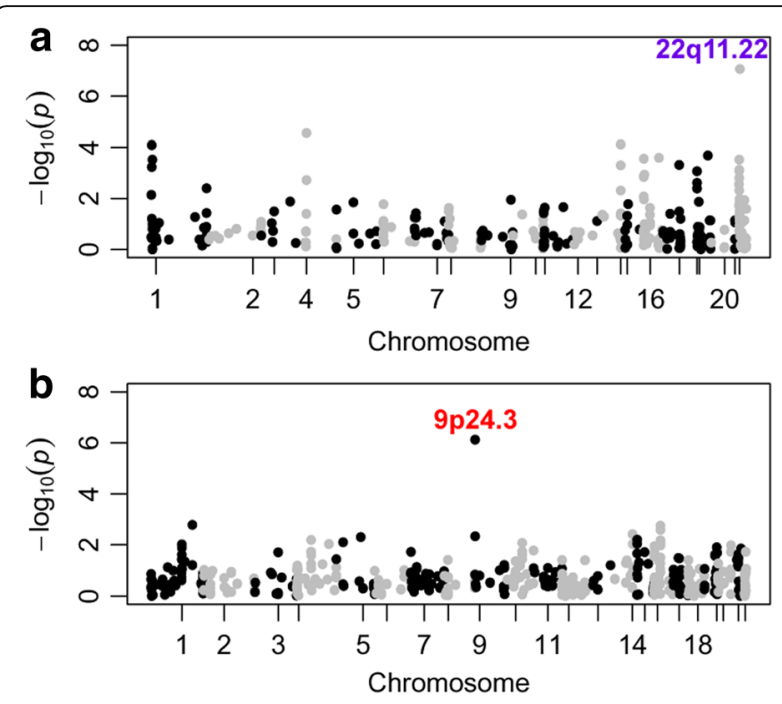

Fig. 1 Manhattan plots for gene-based CNV meta-analysis. The results for deletion CNVs are shown in a and those for duplication CNVs are shown in $\mathbf{b}$. The $-\log 10(P$ value) of each gene ( $y$-axis) in the meta-analysis is plotted against the genomic position ( $x$-axis). Significant loci are indicated on the plot. The locus of 22q11.22 is a known locus for neuropsychiatric disorders and 9p24.3 is a novel locus at which each CNV carrier has been validated by manual visual review of BAF and LRR plots and GPCR experiments

specifically conducted validation for $\mathrm{CNV}$ carriers in this cohort by two approaches. First, we manually examined the CNV calls by visually reviewing the raw intensity and genotype values for probes in this region and flanking regions (Additional file 2: Figure S3). We have previously reported that visually validated $\mathrm{CNVs}$ yield experimental validation success rates above 95\% [32]. For the CNVs contributing calls in this region, we observed a moderate gain in LRR intensity and alternative banding of BAF at 0.33 and 0.66 instead of 0.5 , which are indicative of clear duplication CNV signals. Second, we also tested the CNV status of this region using an independent experimental approach, the qPCR assay, on the above samples (Table 4). We included one sample from our cohort without a CNV in this region as a negative control. Each sample containing a duplication in the DOCK8-KANK1 region was tested by five $\mathrm{qPCR}$ assays. The results showed that the CNV in each of the ten samples was also detected by the corresponding qPCR assays, and the $\mathrm{CN}=2$ control sample did not show any $\mathrm{CNV}$ in all qPCR assays across this region. There were a few regions flanking the CNVs detected by arrays where duplications were observed with qPCR, refining the CNV boundaries. Thus, the CNV data from the arrays were validated by both an in silico approach and an independent experimental assay. An inconsistency between the array and qPCR results was seen for sample S9 with probe 141, and the array result for this sample is likely due to ambiguity in CNV boundary determination rather than presence vs. absence based on CNV calling from SNP arrays.

Each of the contributing CNVs overlapping regions from the five cohorts overlaps with DOCK 8 exons, which is likely to have an impact on DOCK8 expression. In addition, assessing the annotations in ENCODE [45] and ROADMAP [46] databases, we found that the DOCK8 overlapping CNV region from each cohort contains many histone marks and DNase sites (Additional file 1: Table S2). Further interrogating these regions in two eQTL databases-GTEx [47] and GRASP [48]-we observed significant brain tissue eQTL SNPs in DOCK8 overlapping the CNV region in four out of the five cohorts (Additional file 1: Table S2). For the significant eQTLs, the genes regulated include CBWD1, DMRT3, DOCK8, FOXD4, and KANK1, all of which are located in the same topologically associating domains (TADs; Additional file 2: Figure S4), suggesting that the expression of these genes may also be affected.

\section{Discussion}

Cumulative evidence indicates a shared genetic etiology of neurodevelopmental and neuropsychiatric diseases. We performed CNV meta-analysis in five major neurodevelopmental/psychiatric diseases. Using gene-based association statistics, we robustly meta-analyzed different psychiatric conditions across distinct microarrays. This is the first large-scale CNV meta-analysis across a spectrum of neuropsychiatric disorders. We identified the DOCK8/KANK1 locus as containing exonic CNVs with genome-wide significant meta $P$ values and consistent direction of effects across all five cohorts. The highly significant associations of DOCK8/KANK1 duplications

Table 3 Contributing signals for the DOCK8 gene from each psychiatric disease cohort

\begin{tabular}{lllllll}
\hline Disease cohort & Overlapping CNV region (hg18) & CNV type & Odds ratio & $P$ value & Number of case CNVs (\%) & Number of control CNVs (\%) \\
\hline Janssen SCZ and BD & Chr9:396118-474850 & Dup & Infinity & 0.00693 & $6(0.21)$ & $0(0)$ \\
CHOP SCZ & Chr9:372245-389052 & Dup & 10.71 & 0.008 & $7(0.73)$ & $1(0.07)$ \\
CHOP ASD & Chr9:407918-474786 & Dup & Infinity & 0.00384 & $7(0.34)$ & $0(0)$ \\
CHOP ADHD & Chr9: 293639-352917 & Dup & 3.32 & 0.0899 & $4(0.32)$ & $4(0.10)$ \\
Depression & Chr9: $283360-294957$ & Dup & 4.96 & 0.00731 & $8(1.24)$ & $4(0.25)$ \\
\hline
\end{tabular}

Number of case CNVs is the number of cases having a DOCK8 duplication and the frequency among all cases. Number of control CNVs is the number of controls having a DOCK8 duplication CNV and the frequency among all controls 


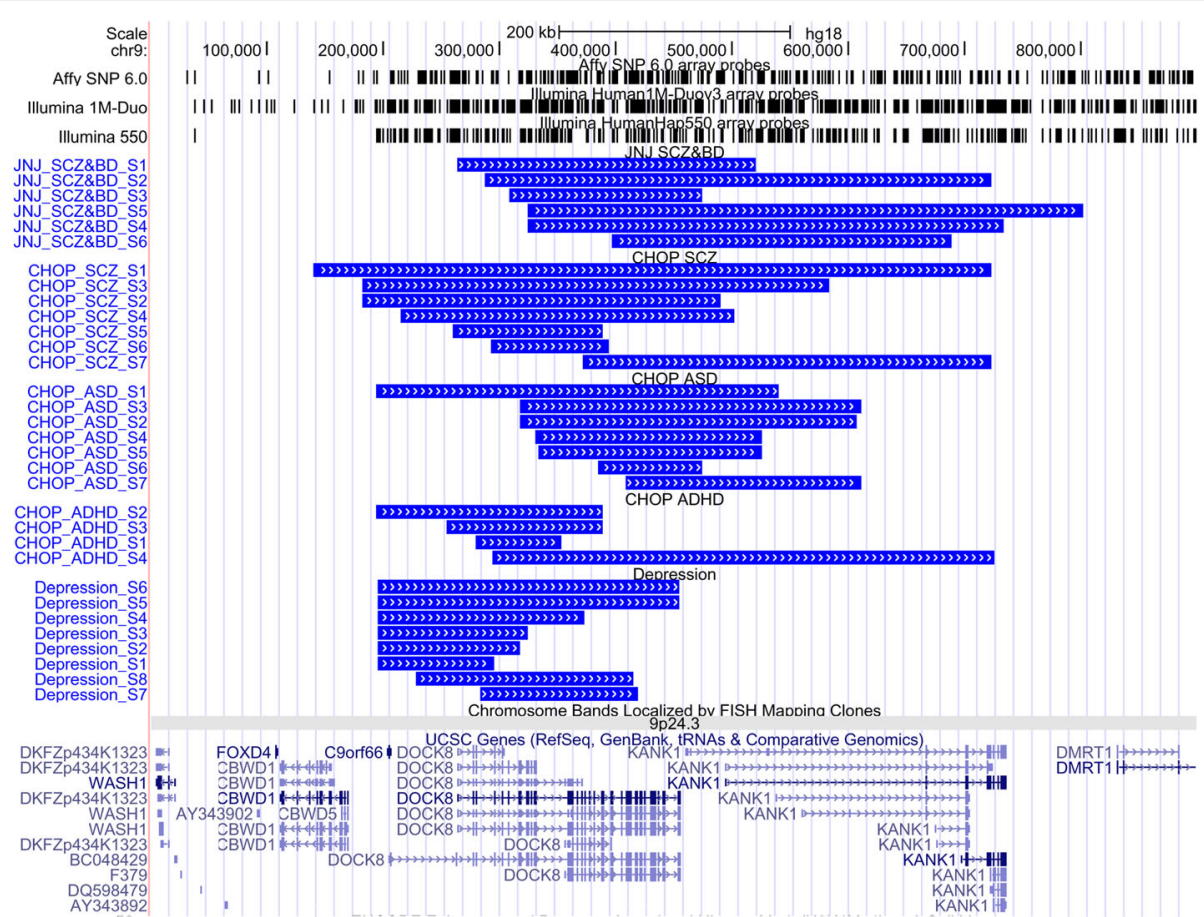

Fig. 2 Contributing calls in the DOCK8/KANK1 region from each cohort. Black bars indicate the SNP coverage of each genotyping array and blue rectangles represent each individual duplication call observed among neuropsychiatric cases in each cohort

were further validated by an independent experimental approach. The identification of shared structural variants underlying the five neuropsychiatric disorders helps to refine the genetic basis for co-morbidity and cooccurrence of neuropsychiatric diseases among individuals or families has the potential to help in the development of common therapeutics of shared genetic targets across different diseases.

The duplications of DOCK8 and KANK1 at 9p24.3 are intriguing given that these genes have been shown to be involved in neurodevelopment and neurological functions. DOCK8 is the dedicator of cytokinesis 8 , a

Table 4 qPCR validation of duplications in the DOCK8-KANK1 region

\begin{tabular}{|l|l|l|l|l|l|l|}
\hline & & Assay132 & Assay134 & Assay135 & Assay137 & Assay141 \\
\hline Sample & CNV & $\begin{array}{l}\text { chr9:279,035- } \\
279138\end{array}$ & $\begin{array}{l}\text { chr9:326,544- } \\
326608\end{array}$ & $\begin{array}{l}\text { chr9:416,891- } \\
417000\end{array}$ & $\begin{array}{l}\text { chr9:460,349- } \\
460443\end{array}$ & $\begin{array}{l}\text { chr9:537,138- } \\
537211\end{array}$ \\
\hline S1 & $\begin{array}{l}\text { chr9:263161- } \\
520703\end{array}$ & 3 & 3 & 3 & 3 & 2 \\
\hline S2 & $\begin{array}{l}\text { chr9:287395- } \\
723374\end{array}$ & 2 & 3 & 3 & 3 & 3 \\
\hline S3 & $\begin{array}{l}\text { chr9:308154- } \\
474850\end{array}$ & 2 & 3 & 3 & 3 & 2 \\
\hline S4 & $\begin{array}{l}\text { chr9:323820- } \\
733353\end{array}$ & 2 & 3 & 3 & 3 & 3 \\
\hline S5 & $\begin{array}{l}\text { chr9:323820- } \\
801972\end{array}$ & 2 & 3 & 3 & 3 & 4 \\
\hline S6 & $\begin{array}{l}\text { chr9:396118- } \\
689065\end{array}$ & 2 & 2 & 3 & 3 & 3 \\
\hline S7 & $\begin{array}{l}\text { chr9:468154- } \\
697859\end{array}$ & 2 & 2 & 3 & 3 & 3 \\
\hline S8 & $\begin{array}{l}\text { chr9:474299- } \\
702599\end{array}$ & 2 & 2 & 3 & 3 & 3 \\
\hline S9 & $\begin{array}{l}c h r 9: 490811- \\
534956\end{array}$ & 2 & 2 & 3 & 3 & 2 \\
\hline S10 & $\begin{array}{l}\text { chr9:516773- } \\
801972\end{array}$ & 2 & 2 & 2 & 2 & 3 \\
\hline Con & & 2 & 2 & 2 & 2 & 2 \\
\hline
\end{tabular}

Each sample was tested by five qPCR assays which cover much of the duplication regions. Each result represents copy number calculated from triplicate runs. The table has been shaded gray for assays that were within the duplication call for that subject detected by arrays, and the CNVs validated by qPCR are highlighted in red 
member of the DOCK180 family of guanine nucleotide exchange factors (GEF), which includes 11 DOCK genes $[49,50]$. GEF proteins are important components of intracellular signaling networks, activating small GTPases by exchanging bound GDP for free GTP [51]. DOCK8 is expressed in adult and fetal brain tissues and deletion or translocation breakpoints that disrupt its function have been found in individual patients with intellectual disability [52]. This is the first time that DOCK8 duplications were found to be significantly associated with a spectrum of neurospsychiatric disorders, suggesting that a tightly regulated DOCK 8 expression level may be required for normal cellular function. The neighboring gene of DOCK8 is KANK1 (KN motif and ankyrin repeat domains 1 ), which has also been demonstrated to play a role in neuronal functions. KANK1 functions in actin cytoskeleton formation by competing for 14-3-3 binding upon phosphorylation by Akt and inhibiting RhoA activity [53, 54]. It inhibits neurite outgrowth, actin fiber formation, and cell migration, depending on the competitive interaction with BAIAP2 to block its association with activated RAC1 [55]. KANK1 functions in regulating microtubule dynamics at the cell cortex by recruiting KIF21A, which is important in neuronal development $[56,57]$. Mutations in this gene cause cerebral palsy spastic quadriplegic type 2, a central nervous system development disorder [58]. In the DECIPHER dataset, 16 subjects with intellectual disability carry DOCK8 duplications/gains and ten carry KANK1 duplications/gains [59].

The novelty of our finding lies in the illustration of the significant association of DOCK8/KANK1 with multiple neuropsychiatric diseases. In the $\mathrm{CNV}$ study of Coe et al. [60] for intellectual disability, developmental delay, and/or ASD, DOCK8 is nominally significant for deletions $(P=0.000281)$ but not duplications. In the recent $\mathrm{CNV}$ study of schizophrenia by Marshall et al. [42], DMRT1, which is at the same cytoband of $9 \mathrm{p} 24.3$, was reported to be a novel CNV-containing gene significantly associated with schizophrenia in the test for a combined (deletion + duplication) $\mathrm{CNV}$ analysis. The reported DMRT1 CNV locus (chr9:831690-959090, hg18) is more than $400 \mathrm{~kb}$ downstream of the DOCK8/KANK1 region found in our study. In addition, the DOCK8/KANK1 region is not highly prone to $\mathrm{CNV}$ in the general population. In our study, $0.25 \%$ or less of the control subjects carry DOCK8/KANK1 duplications in each cohort. The Database of Genomic Variants (DGV) [61] shows 65 duplications in the DOCK8 gene. Among them, 33 were reported in $\mathrm{CNV}$ studies of developmental delay $[60,62]$, and 32 duplications in DOCK8 were reported from other $\mathrm{CNV}$ population studies. This is also similar to another gene, NRXN1, the deletion of which is significantly associated with schizophrenia [63]. A total of 144 deletions/loss were reported in DGV for the NRXN1 gene, among which 45 were reported in the CNV studies of developmental delay and the remaining 99 were from other CNV population studies. Therefore, the significant association in the meta-analysis reflects its potential contribution to the pathology of neurodevelopmental/ psychiatric disorders. In addition, the novelty of our finding lies in the identification of the significant association of DOCK8 duplication with multiple neurodevelopmental/psychiatric disorders across cohorts (association $P<0.01$ for four out of five cohorts), implicating its common role as a risk locus for these diseases.

To compensate for the incomplete overlapping in SNP coverage between study cohorts, we conducted gene-based meta-analysis, which has been widely adopted in both common and rare variant analyses. One caveat is that $\mathrm{CNV}$ overlaps with different gene regions might not have the same effect on the expression of the gene. Some may result in frame shifts and the complete loss of function of the gene and some may have minor effects on the gene. Nevertheless, the biological function of each gene is maintained in a fine balanced state for cellular activities. Even minor perturbation of its expression could lead to pathological consequences. The discovery of CNVs in DOCK 8 and KANK1 across all five diseases not only adds to the growing catalog of neurodevelopmental variants but also paves the way for new diagnostics opportunities and interventions which could be applied across multiple clinical indications. However, functional studies are needed to better understand the biological effect of these variations.

\section{Conclusions}

With the growing awareness of the high impact of childhood psychiatric conditions comes the important need for large-scale genetic studies and a unified picture of the catalog of rare variants underlying these conditions. We have undertaken the unprecedented step to meta-analyze CNVs across five neurodevelopmental/psychiatric diseases and have uncovered significant structural variation at the DOCK8/KANK1 locus shared by these diseases, emphasizing the common genetic component involved in the pathogenesis of neuropsychiatric disorders.

\section{Additional files}

Additional file 1: Table S1. The quality control criteria based on CNV metrics applied to each cohort. Table S2. The number of histone marks, DNase, and eQTLs in each DOCK8 CNV. (PDF $127 \mathrm{~kb}$ ) 
Additional file 2: Figure S1. Circular plot showing the intersections of genes harboring case-enriched CNVs between the five neuropsychiatric cohorts. Figure S2. The BAF and LRR plots of ZNF280A deletions. Figure S3. The BAF and LRR plots of DOCK8/KANK1 duplications. Figure S4. The topologically associating domains (TAD) at the chromosome 9 DOCK8/ KANK1 region. (PDF $2076 \mathrm{~kb})$

\section{Abbreviations}

ADHD: Attention deficit hyperactivity disorder; AGP: Autism Genome Project; AGRE: Autism Genetics Resource Exchange; ASD: Autism spectrum disorders; BAF: B allele frequency; BD: Bipolar disease; CAG: Center for Applied Genomics; CHOP: the Children's Hospital of Philadelphia; CNV: Copy number variation; GAIN: Genetic Association Information Network; GCWF: GC base pair wave factor; GEF: Guanine nucleotide exchange factor; GWAS: Genomewide association studies; KANK1: KN motif and ankyrin repeat domains 1; LMM: Linear mixed model; LRR: Log R ratio; MDD: Major depressive disorder; NESDA: Netherlands Study of Depression and Anxiety; PCA: Principal component analysis; QC: Quality control; qPCR: Quantitative polymerase chain reaction; SCZ: Schizophrenia; SNP: Single nucleotide polymorphism; TAD: Topologically associating domains; UPL: Universal Probe Library

\section{Acknowledgments}

We thank all the study volunteers for participating in the research studies and the clinicians and support staff for enabling patient recruitment and blood sample collection. We also thank the staff in the Neuroscience Biomarkers Genomic Lab at Janssen \& the Center for Applied Genomics at CHOP for sample processing and the staff at Illumina for genotyping Janssen DNA samples. We thank Anthony Santos, Nicole Bottrel, Monique-Andree Franc, and William Cafferty of Janssen Research \& Development for operational support, and Stefanie Rassnick of Janssen Research \& Development for input in setting up the collaboration and for discussion. Members of the Janssen-CHOP Neuropsychiatric Genomics Working Group: CHOP, Patrick Sleiman 1,2, Joseph Glessner', Dexter Hadley', Charlly Kao', Zhiliang $\mathrm{Wu}^{1}$, Cecilia Kim', Kelly Thomas', Hakon Hakonarson ',2, Janssen, Dai Wang ${ }^{3}$, Reyna Favis ${ }^{3}$, Dong-Jing Fu ${ }^{4}$, Hedy Chung ${ }^{4}$, Adam Savitz ${ }^{5}$, Srihari $\mathrm{Gopal}^{5}$, Nadine Cohen ${ }^{3}$, Qingqin $\mathrm{Li}^{5}$.

'The Center for Applied Genomics, The Children's Hospital of Philadelphia, Philadelphia, PA 19104, USA; ${ }^{2}$ Department of Pediatrics, University of Pennsylvania School of Medicine, Philadelphia, PA 19104, USA; ${ }^{3}$ Janssen Research \& Development, LLC, Raritan, NJ, 08869, USA; ${ }^{4}$ Janssen Scientific Affairs, LLC, Titusville, NJ, 08540, USA; ${ }^{5}$ Janssen Research \& Development, LLC, Titusville, NJ, 08869, USA.

\section{Funding}

Funding for this study was provided by an Institutional Development Award to the Center for Applied Genomics from The Children's Hospital of Philadelphia; Adele and Daniel Kubert donation, University of Pennsylvania Biomedical Graduate Studies training grant; the Cotswold foundation; by 1R01MH097284-01 from the National Institute of Mental Health (NIMH), and sponsored research to The Children's Hospital of Philadelphia by Janssen Research \& Development, LLC.

\section{Availability of data and materials}

The dbGaP study accessions: CHOP SCZ, phs000167.v1.p1 and phs000021.v3.p2; CHOP ASD, phs000267.v5.p2; CHOP ADHD, phs000016.v2.p2; depression, phs000020.v2.p1. The other datasets used and/ or analyzed during the current study are available from the corresponding author on reasonable request.

\section{Authors' contributions}

$\mathrm{HH}$ and $\mathrm{NC}$ conceived the project and HH supervised the study. QL managed the Janssen samples and genetic data. JG and JL performed the analysis with the help of JG, AD, LL, and DH. DW performed Janssen genotype data QC. REG assisted with phenotyping of the PENN cohort. MM conducted qPCR validation. JG, JL, AD, and HH wrote the manuscript. The Janssen-CHOP Neuropsychiatric Genomics Working Group collected samples and performed phenotype classification. All authors contributed to the data analysis review, discussions, and contributed to the final manuscript. All authors read and approved the final manuscript.

\section{Ethics approval and consent to participate}

All cohorts in our study were from published datasets. Study protocols used in each cohort have been approved by corresponding Institutional Review Board or equivalent committees, and written informed consent was given from each participant or parent. Our research complies with the Declaration of Helsinki.

\section{Consent for publication}

Not applicable.

\section{Competing interests}

Dr. Nadine Cohen is a former employee of Janssen Research \& Development Drs. Dai Wang and Qingqin Li are current employees of Janssen Research \& Development. The remaining authors declare that they have no competing interests. Funding for this study included sponsored research to The Children's Hospital of Philadelphia by Janssen Research \& Development, LLC.

\section{Publisher's Note}

Springer Nature remains neutral with regard to jurisdictional claims in published maps and institutional affiliations.

\section{Author details}

'The Center for Applied Genomics, Abramson Research Center, Children's Hospital of Philadelphia, Philadelphia, PA 19104, USA. ${ }^{2}$ Department of Cell Biology, Tianjin Medical University, Tianjin, China. ${ }^{3}$ Janssen Research \& Development, LLC, Raritan, NJ 08869, USA. ${ }^{4}$ Department of Psychiatry, Perelman School of Medicine, University of Pennsylvania, Philadelphia, PA 19104, USA. ${ }^{5}$ Department of Pediatrics, Perelman School of Medicine, University of Pennsylvania, Philadelphia, PA 19104, USA. ${ }^{6}$ Division of Human Genetics, Children's Hospital of Philadelphia, Philadelphia, PA 19104, USA. ${ }^{7}$ Janssen Research \& Development, LLC, Titusville, NJ 08560, USA.

Received: 13 July 2017 Accepted: 14 November 2017 Published online: 30 November 2017

\section{References}

1. Cross-Disorder Group of the Psychiatric Genomics C. Identification of risk loci with shared effects on five major psychiatric disorders: a genome-wide analysis. Lancet. 2013;381(9875):1371-9.

2. International Schizophrenia Consortium, Purcell SM, Wray NR, Stone $L_{\text {, }}$ Visscher PM, O'Donovan MC, Sullivan PF, Sklar P. Common polygenic variation contributes to risk of schizophrenia and bipolar disorder. Nature. 2009;460(7256):748-52.

3. Cross-Disorder Group of the Psychiatric Genomics Consortium, Lee SH, Ripke S, Neale BM, Faraone SV, Purcell SM, Perlis RH, Mowry BJ, Thapar A, Goddard ME, et al. Genetic relationship between five psychiatric disorders estimated from genome-wide SNPs. Nat Genet. 2013;45(9):984-94.

4. Glessner JT, Reilly MP, Kim CE, Takahashi N, Albano A, Hou C, Bradfield JP, Zhang $\mathrm{H}$, Sleiman PM, Flory JH, et al. Strong synaptic transmission impact by copy number variations in schizophrenia. Proc Natl Acad Sci U S A. 2010; 107(23):10584-9.

5. Glessner JT, Wang K, Cai G, Korvatska O, Kim CE, Wood S, Zhang H, Estes A, Brune CW, Bradfield JP, et al. Autism genome-wide copy number variation reveals ubiquitin and neuronal genes. Nature. 2009;459(7246):569-73.

6. Glessner JT, Wang K, Sleiman PM, Zhang H, Kim CE, Flory JH, Bradfield JP, Imielinski M, Frackelton EC, Qiu H, et al. Duplication of the SLIT3 locus on 5q35.1 predisposes to major depressive disorder. PLoS One. 2010;5(12): e15463.

7. Elia J, Glessner JT, Wang K, Takahashi N, Shtir CJ, Hadley D, Sleiman PM, Zhang H, Kim CE, Robison R, et al. Genome-wide copy number variation study associates metabotropic glutamate receptor gene networks with attention deficit hyperactivity disorder. Nat Genet. 2012;44(1):78-84.

8. Schizophrenia Psychiatric Genome-Wide Association Study Consortium. Genome-wide association study identifies five new schizophrenia loci. Nat Genet. 2011;43(10):969-76.

9. Psychiatric GWAS Consortium Bipolar Disorder Working Group. Large-scale genome-wide association analysis of bipolar disorder identifies a new susceptibility locus near ODZ4. Nat Genet. 2011;43(10):977-83.

10. Sleiman P, Wang D, Glessner J, Hadley D, Gur RE, Cohen N, Li Q, Hakonarson $\mathrm{H}$, Janssen CNGWG. GWAS meta analysis identifies TSNARE1 as a novel Schizophrenia/Bipolar susceptibility locus. Sci Rep. 2013;3:3075. 
11. Vissers LE, Gilissen C, Veltman JA. Genetic studies in intellectual disability and related disorders. Nat Rev Genet. 2016;17(1):9-18.

12. Xu B, Roos JL, Dexheimer P, Boone B, Plummer B, Levy S, Gogos JA, Karayiorgou $\mathrm{M}$. Exome sequencing supports a de novo mutational paradigm for schizophrenia. Nat Genet. 2011;43(9):864-8.

13. Gulsuner S, Walsh T, Watts AC, Lee MK, Thornton AM, Casadei S, Rippey C, Shahin H, Consortium on the Genetics of S, Group PS, et al. Spatial and temporal mapping of de novo mutations in schizophrenia to a fetal prefrontal cortical network. Cell. 2013;154(3):518-29.

14. Fromer M, Pocklington AJ, Kavanagh DH, Williams HJ, Dwyer S, Gormley P, Georgieva L, Rees E, Palta P, Ruderfer DM, et al. De novo mutations in schizophrenia implicate synaptic networks. Nature. 2014; 506(7487):179-84

15. CONVERGE consortium. Sparse whole-genome sequencing identifies two loci for major depressive disorder. Nature. 2015;523(7562):588-91.

16. Hyde CL, Nagle MW, Tian C, Chen X, Paciga SA, Wendland JR, Tung JY, Hinds DA, Perlis RH, Winslow AR. Identification of 15 genetic loci associated with risk of major depression in individuals of European descent. Nat Genet. 2016;48(9):1031-6.

17. Okbay A, Baselmans BM, De Neve JE, Turley P, Nivard MG, Fontana MA, Meddens SF, Linner RK, Rietveld CA, Derringer J, et al. Genetic variants associated with subjective well-being, depressive symptoms, and neuroticism identified through genome-wide analyses. Nat Genet. 2016: 48(6):624-33.

18. Direk N, Williams S, Smith JA, Ripke S, Air T, Amare AT, Amin N, Baune BT, Bennett DA, Blackwood DHR, et al. An analysis of two genome-wide association meta-analyses identifies a new locus for broad depression phenotype. Biol Psychiatry. 2017;82(5):322-9.

19. Mullins N, Lewis CM. Genetics of depression: progress at last. Curr Psychiatry Rep. 2017;19(8):43

20. MacArthur J, Bowler E, Cerezo M, Gil L, Hall P, Hastings E, Junkins $H$, McMahon A, Milano A, Morales J, et al. The new NHGRI-EBI Catalog of published genome-wide association studies (GWAS Catalog). Nucleic Acids Res. 2017;45(D1):D896-901.

21. Hadley D, Wu ZL, Kao C, Kini A, Mohamed-Hadley A, Thomas K, Vazquez L, Qiu H, Mentch F, Pellegrino R, et al. The impact of the metabotropic glutamate receptor and other gene family interaction networks on autism. Nat Commun. 2014:5:4074.

22. Green EK, Rees E, Walters JT, Smith KG, Forty L, Grozeva D, Moran JL, Sklar P, Ripke S, Chambert KD, et al. Copy number variation in bipolar disorder. Mol Psychiatry. 2016;21(1):89-93.

23. Szatkiewicz JP, O'Dushlaine C, Chen G, Chambert K, Moran JL, Neale BM, Fromer M, Ruderfer D, Akterin S, Bergen SE, et al. Copy number variation in schizophrenia in Sweden. Mol Psychiatry. 2014;19(7):762-73.

24. Schneider M, Debbane M, Bassett AS, Chow EW, Fung WL, van den Bree $M$, Owen M, Murphy KC, Niarchou M, Kates WR, et al. Psychiatric disorders from childhood to adulthood in 22q11.2 deletion syndrome: results from the International Consortium on Brain and Behavior in 22q11.2 Deletion Syndrome. Am J Psychiatry. 2014;171(6):627-39.

25. Hassfurther A, Komini E, Fischer J, Leipoldt M. Clinical and genetic heterogeneity of the 15q13.3 microdeletion syndrome. Mol Syndromol. 2016;6(5):222-8

26. Ching MS, Shen Y, Tan WH, Jeste SS, Morrow EM, Chen X, Mukaddes NM, Yoo SY, Hanson E, Hundley R, et al. Deletions of NRXN1 (neurexin-1) predispose to a wide spectrum of developmental disorders. Am J Med Genet B Neuropsychiatr Genet. 2010;153B(4):937-47.

27. Schizophrenia Working Group of the Psychiatric Genomics C. Biological insights from 108 schizophrenia-associated genetic loci. Nature. 2014; 511(7510):421-7.

28. Boomsma DI, Willemsen G, Sullivan PF, Heutink P, Meijer P, Sondervan D, Kluft C, Smit G, Nolen WA, Zitman FG, et al. Genome-wide association of major depression: description of samples for the GAIN Major Depressive Disorder Study: NTR and NESDA biobank projects. Eur J Hum Genet. 2008; 16(3):335-42.

29. Price AL, Patterson NJ, Plenge RM, Weinblatt ME, Shadick NA, Reich D. Principal components analysis corrects for stratification in genome-wide association studies. Nat Genet. 2006;38(8):904-9.

30. Wang K, Li M, Hadley D, Liu R, Glessner J, Grant SF, Hakonarson H, Bucan M. PennCNV: an integrated hidden Markov model designed for high-resolution copy number variation detection in whole-genome SNP genotyping data. Genome Res. 2007;17(11):1665-74.
31. Purcell S, Neale B, Todd-Brown K, Thomas L, Ferreira MA, Bender D, Maller J, Sklar P, de Bakker PI, Daly MJ, et al. PLINK: a tool set for whole-genome association and population-based linkage analyses. Am J Hum Genet. 2007:81(3):559-75.

32. Glessner JT, Li J, Hakonarson H. ParseCNV integrative copy number variation association software with quality tracking. Nucleic Acids Res. 2013;41(5):e64.

33. Zhou $X$, Stephens M. Efficient multivariate linear mixed model algorithms for genome-wide association studies. Nat Methods. 2014 11(4):407-9.

34. Willer CJ, Li Y, Abecasis GR. METAL: fast and efficient meta-analysis of genomewide association scans. Bioinformatics. 2010;26(17):2190-1.

35. Wang M, Zhao Y, Zhang B. Efficient test and visualization of multi-set intersections. Sci Rep. 2015;5:16923.

36. Li YR, Li J, Zhao SD, Bradfield JP, Mentch FD, Maggadottir SM, Hou C, Abrams DJ, Chang D, Gao F, et al. Meta-analysis of shared genetic architecture across ten pediatric autoimmune diseases. Nat Med. 2015; 21(9):1018-27.

37. Gamazon ER, Wheeler HE, Shah KP, Mozaffari SV, Aquino-Michaels K Carroll RJ, Eyler AE, Denny JC, Consortium GT, Nicolae DL, et al. A gene-based association method for mapping traits using reference transcriptome data. Nat Genet. 2015;47(9):1091-8.

38. Liu JZ, McRae AF, Nyholt DR, Medland SE, Wray NR, Brown KM, Investigators A, Hayward NK, Montgomery GW, Visscher PM, et al. A versatile gene-based test for genome-wide association studies. Am J Hum Genet. 2010;87(1):139-45.

39. Moutsianas L, Agarwala V, Fuchsberger C, Flannick J, Rivas MA, Gaulton KJ, Albers PK, Go TDC, McVean G, Boehnke M, et al. The power of gene-based rare variant methods to detect disease-associated variation and test hypotheses about complex disease. PLoS Genet. 2015;11(4): e1005165.

40. Wang K, Li M, Hakonarson $\mathrm{H}$. Analysing biological pathways in genome-wide association studies. Nat Rev Genet. 2010;11(12):843-54.

41. Scott RA, Lagou V, Welch RP, Wheeler E, Montasser ME, Luan J, Magi R, Strawbridge RJ, Rehnberg E, Gustafsson S, et al. Large-scale association analyses identify new loci influencing glycemic traits and provide insight into the underlying biological pathways. Nat Genet. 2012;44(9):991-1005.

42. Marshall CR, Howrigan DP, Merico D, Thiruvahindrapuram B, Wu W, Greer DS, Antaki D, Shetty A, Holmans PA, Pinto D, et al. Contribution of copy number variants to schizophrenia from a genome-wide study of 41,321 subjects. Nat Genet. 2017:49(1):27-35

43. Rees E, Kirov G, Sanders A, Walters JT, Chambert KD, Shi J, Szatkiewicz J, O'Dushlaine C, Richards AL, Green EK, et al. Evidence that duplications of 22q11.2 protect against schizophrenia. Mol Psychiatry. 2014;19(1):37-40.

44. Chen J, Calhoun VD, Perrone-Bizzozero NI, Pearlson GD, Sui J, Du Y, Liu J. A pilot study on commonality and specificity of copy number variants in schizophrenia and bipolar disorder. Transl Psychiatry. 2016;6(5):e824

45. ENCODE Project Consortium. An integrated encyclopedia of DNA elements in the human genome. Nature. 2012;489(7414):57-74.

46. Roadmap Epigenomics Consortium, Kundaje A, Meuleman W, Ernst J, Bilenky M, Yen A, Heravi-Moussavi A, Kheradpour P, Zhang Z, Wang J, et al. Integrative analysis of 111 reference human epigenomes. Nature. 2015:518(7539):317-30.

47. GTEx Consortium. Human genomics. The Genotype-Tissue Expression (GTEx) pilot analysis: multitissue gene regulation in humans. Science. 2015; 348(6235):648-60

48. Leslie R, O'Donnell CJ, Johnson AD. GRASP: analysis of genotypephenotype results from 1390 genome-wide association studies and corresponding open access database. Bioinformatics. 2014;30(12): i185-94.

49. Ruusala A, Aspenstrom P. Isolation and characterisation of DOCK8, a member of the DOCK180-related regulators of cell morphology. FEBS Lett. 2004;572(1-3):159-66.

50. Su HC. Dedicator of cytokinesis 8 (DOCK8) deficiency. Curr Opin Allergy Clin Immunol. 2010;10(6):515-20.

51. Rossman $\mathrm{KL}$, Der CJ, Sondek J. GEF means go: turning on RHO GTPases with guanine nucleotide-exchange factors. Nat Rev Mol Cell Biol. 2005; 6(2):167-80.

52. Griggs BL, Ladd S, Saul RA, DuPont BR, Srivastava AK. Dedicator of cytokinesis 8 is disrupted in two patients with mental retardation and developmental disabilities. Genomics. 2008;91(2):195-202. 
53. Kakinuma N, Roy BC, Zhu Y, Wang Y, Kiyama R. Kank regulates RhoAdependent formation of actin stress fibers and cell migration via 14-3-3 in PI3K-Akt signaling. J Cell Biol. 2008;181(3):537-49.

54. Kakinuma N, Zhu Y, Wang Y, Roy BC, Kiyama R. Kank proteins: structure, functions and diseases. Cell Mol Life Sci. 2009;66(16):2651-9.

55. Roy BC, Kakinuma N, Kiyama R. Kank attenuates actin remodeling by preventing interaction between IRSp53 and Rac1. J Cell Biol. 2009; 184(2):253-67.

56. Kakinuma N, Kiyama R. A major mutation of KIF21A associated with congenital fibrosis of the extraocular muscles type 1 (CFEOM1) enhances translocation of Kank1 to the membrane. Biochem Biophys Res Commun. 2009;386(4):639-44.

57. van der Vaart B, van Riel WE, Doodhi H, Kevenaar JT, Katrukha EA, Gumy L, Bouchet BP, Grigoriev I, Spangler SA, Yu KL, et al. CFEOM1-associated kinesin KIF21A is a cortical microtubule growth inhibitor. Dev Cell. 2013; 27(2):145-60.

58. Lerer I, Sagi M, Meiner V, Cohen T, Zlotogora J, Abeliovich D. Deletion of the ANKRD15 gene at 9p24.3 causes parent-of-origin-dependent inheritance of familial cerebral palsy. Hum Mol Genet. 2005;14(24):3911-20.

59. Firth HV, Richards SM, Bevan AP, Clayton S, Corpas M, Rajan D, Van Vooren S, Moreau Y, Pettett RM, Carter NP. DECIPHER: Database of Chromosomal Imbalance and Phenotype in Humans Using Ensembl Resources. Am J Hum Genet. 2009;84(4):524-33.

60. Coe BP, Witherspoon K, Rosenfeld JA, van Bon BW, Vulto-van Silfhout AT, Bosco P, Friend KL, Baker C, Buono S, Vissers LE, et al. Refining analyses of copy number variation identifies specific genes associated with developmental delay. Nat Genet. 2014;46(10):1063-71.

61. MacDonald JR, Ziman R, Yuen RK, Feuk L, Scherer SW. The Database of Genomic Variants: a curated collection of structural variation in the human genome. Nucleic Acids Res. 2014;42(Database issue):D986-92.

62. Cooper GM, Coe BP, Girirajan S, Rosenfeld JA, Vu TH, Baker C, Williams C, Stalker H, Hamid R, Hannig V, et al. A copy number variation morbidity map of developmental delay. Nat Genet. 2011;43(9):838-46.

63. Kirov G, Rujescu D, Ingason A, Collier DA, O'Donovan MC, Owen MJ. Neurexin 1 (NRXN1) deletions in schizophrenia. Schizophr Bull. 2009; 35(5):851-4

\section{Submit your next manuscript to BioMed Central and we will help you at every step:}

- We accept pre-submission inquiries

- Our selector tool helps you to find the most relevant journal

- We provide round the clock customer support

- Convenient online submission

- Thorough peer review

- Inclusion in PubMed and all major indexing services

- Maximum visibility for your research

Submit your manuscript at www.biomedcentral.com/submit

) Biomed Central 